\title{
Falsche Konjekturen Bernhardys zu Suidas.
}

I.

Der einen Ausschnitt aus Damaskios' Leben des Isidoros enthaltende

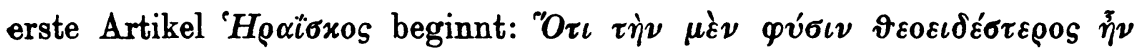

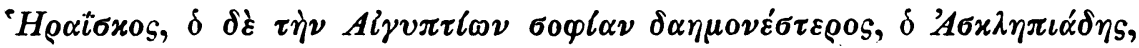

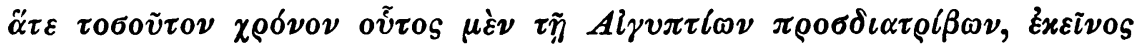

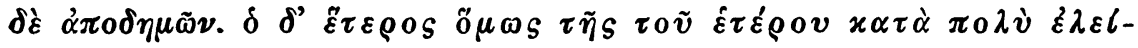

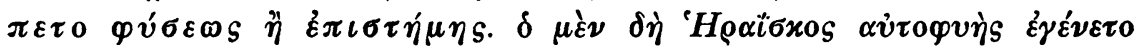

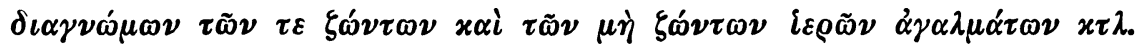
(folgen Beweise für die wunderbare natürlicho Beanlagung des $\mathrm{H}$.). Gegen den SchluB des Artikels (p. 874,8ff. Bernh.) heiBt es: $\delta \delta \dot{\varepsilon}$

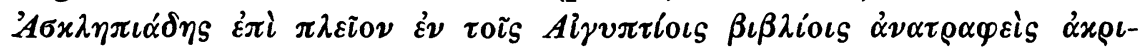

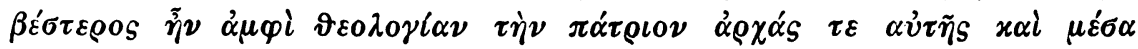

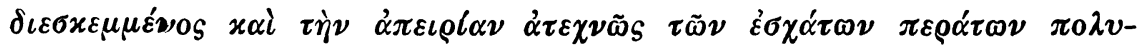

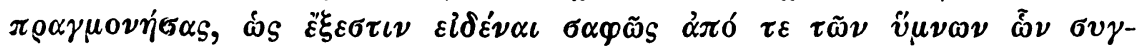

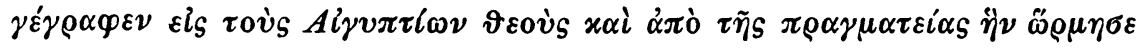

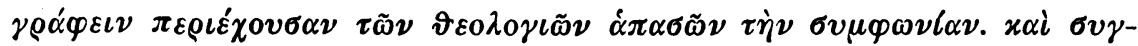

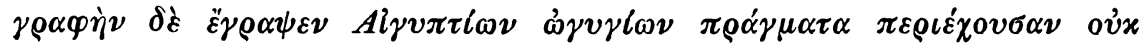

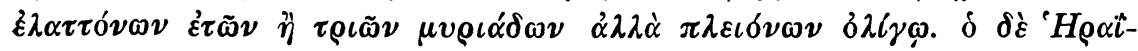

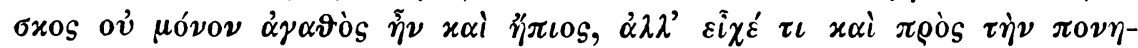

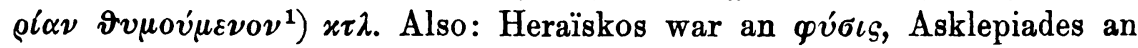
$\dot{\varepsilon} \iota \sigma \tau \eta_{i j u}$ überlegen. Das ist der den ganzen Artikel beherrschende Grundgedanke. Aber - nur das kann der Sinn des gesperrt gedruckten Satzes sein - die Inferiorität des A. hinsiahtlich der qv́бıs war weit gröBer, als die des H. hinsichtlich der $\dot{\pi} \iota \sigma \tau \eta^{\prime} \mu \eta{ }^{2}$ ) Anzumerken ist in dem Satze nur zweierlei. Erstlich die Auslassung des $\mu \tilde{\alpha} \lambda \lambda$ io $\nu$ (

1) So, nicht $\vartheta v \mu o v ́ \mu \varepsilon v o s$, war jedenfalls die ursprüngliche Lesung. Vgl. auch die Anmerkung Bernhardys, der aber im folgenden nicht hätte vor $\pi \alpha \varrho \varepsilon x \beta \propto i \nu \omega \nu$ haltmachen, sondern auch dieses in $\pi \alpha \rho \varepsilon x \beta \alpha \tilde{i} \nu 0 \nu$ verbessern sollen.

2) Dazu stimmt, daB bei Damasc. dubit. et sol. I p. 324 Ruelle, wo es sich um wissenschaftliche Lehren der beiden handelt, kein qualitativer Unterschied hezvortritt.

3) Der Gebrauch beschränkt sich keineswegs auf die von Kühner-Gerth $§ 540$ Anm. 2 berïcksichtigten Fälle. Vgl. die Beispiele bei Lambertus Bos, Ellipses Graecae 


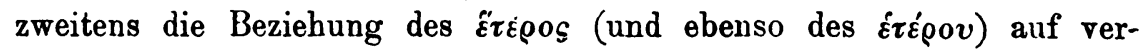
schiedene Personen, die hervortritt, sobald man den Satz vervollständigt:

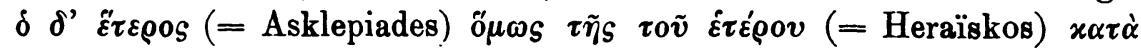

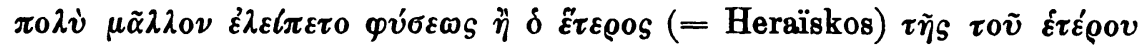
(= Asklepiades) $\dot{\varepsilon} \tau \iota \tau \tau \eta \dot{\mu} \eta$ s. $^{1}$ ) Die Überlieferung deshalb zu beanstanden wäre sehr voreilig. Die gedrängte Kürze hat nun aber Bernhardy dazu geführt, die Stelle gründlich mibzuverstehen und infolgedessen die handschriftliche Lesung im Texte zu ändern. Seine Übersetzung ${ }^{2}$ ) lautet: alter tamen sodali longe erat inferior ingenio vel scientia - wie sinnwidrig, bedarf nach dem oben Gesagten keines Wortes. Im Texte

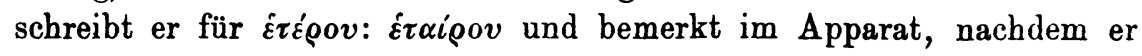

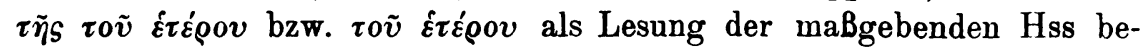
zeichnet hat: Unde promptum fuit elicere, quod posuimus, $\tau \tilde{\eta} s$ rov $\tilde{\varepsilon} \tau \alpha i-$ ○ov. Sed $\tilde{\varepsilon} \tau \varepsilon \rho o S$ abiciendum videtur - beides, sowohl die Änderung von

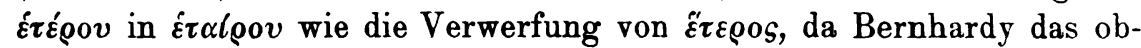
waltende Reziprozitätsverhältnis nicht verstand. Die von Bernhardy zu

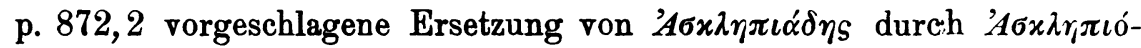

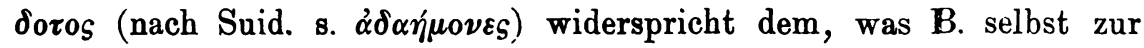
Glosse $\alpha \delta \alpha \eta j \mu \nu \varepsilon s$ und zu unserem Artikel p. 872,5 und 874,15 richtig bemerkt. Die Unterscheidung eines älteren und eines jüngeren Asklepiades bei Damaskios (zu p. 872,5) beruht nur auf Vergewaltigung von Damasc. dub. et sol. I p. $324,3 \mathrm{ff}$. Ru.

II.

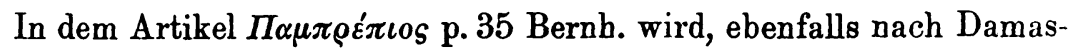
kios' Leben des Isidoros, berichtet, daB die Athener Pamprepios als Lehrer der Grammatik anstellten, und alsdann fortgefahren: ' $O \quad \delta \dot{\varepsilon} \varphi \iota \lambda u^{-}$

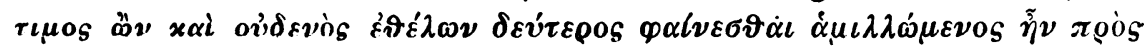

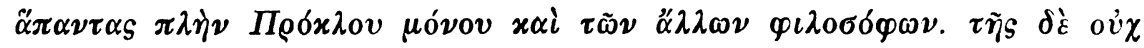

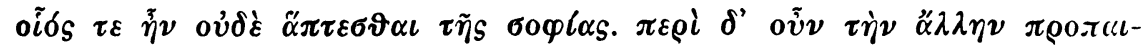

p. 769 ff. ed. Schaefer, K. Krumbacher, Sitzungsber. d. Bayer. Akad., philos.-philol. und hist. Kl. 1896 S. 624 . Auf beider Bemerkungen werde ich aufmerksam durch C. Weyman, Byz. Z. 6 (1897) S. 460. Weitere Beispiele Aristeae epist. § 322; Callinici vit. S. Hypat. p. 70,12. 23.

1) Die Häte hätte sich leicht vermeiden lassen durch folgende Formulie-

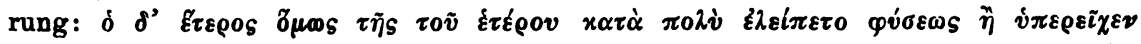

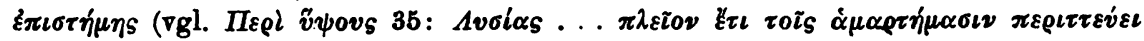
iे $\left.\tau \alpha \tau_{S} \dot{\alpha} \rho \varepsilon \alpha \tau_{S} \lambda \varepsilon i \pi \varepsilon \tau \alpha \iota\right)$.

2) Bernhardy hat die von Küster verbesserte Úbersetzung des Aem. Portus umgearbeitet und trägt für ihre jetzige Gestalt die Verantwortung (vgl. tom. I p. XXIII). DaB er die in Frage kommende Stelle revidiert hat, geht daraus hervor,

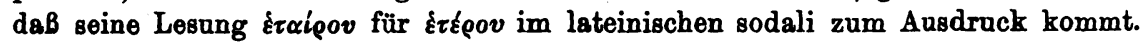




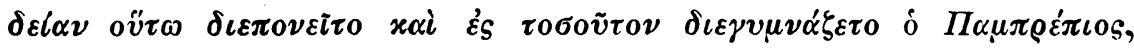

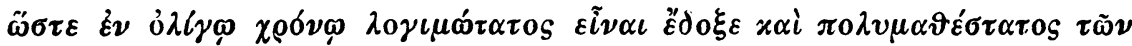

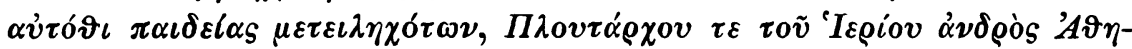

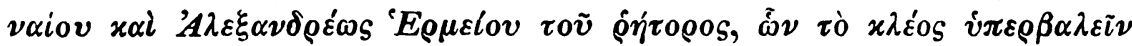

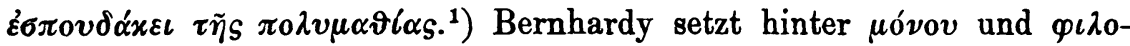

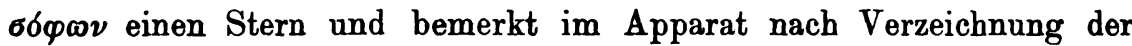

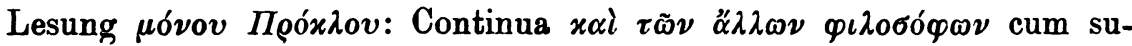
perioribus male cohaerere satis intelligitur .... Iam illa $\tau \tilde{\eta} s \delta \dot{\varepsilon} \ldots .$.

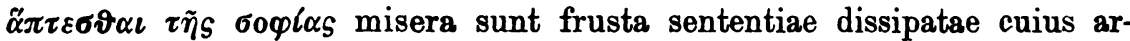
gumentum e sequentibus licet divinare. Nunc indicia lacunae posuimus.

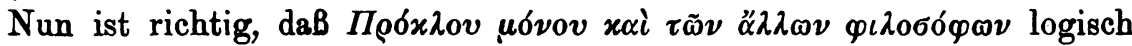
ungereimt ist. Aber psychologisch erklärt sich diese Ausdrucksweise sehr wohl aus der dominierenden Stellung des Proklos unter den gleichzeitigen athenischen Philosophen, der zufolge die anderen gewissermaßen nur anhangsweise erwähnt werden: an Proklos allein wagte sich P. nicht heran, auch nicht an die anderen Philosophen. Am wenigsten ist $\mathrm{zu}$ verstehen, weshalb die Worte $\tau \tilde{\eta} S \delta^{\prime} \dot{\varepsilon} \ldots \sigma o \varphi l \alpha s$ misera frusta sententia dissipatae sein sollen. Allerdings wird man für $\tau \tilde{\eta} s$ zu setzen haben $\tau \alpha{ }^{\prime} \tau \eta$ s. Dann ist aber alles in Ordnung. P. rivalisierte also nicht mitt Proklos und den anderen Philosophen und war überhaupt zur Philossophie nicht befähigt ${ }^{2}$ ), aber auf dem Gebiete der $\pi \rho 0 \pi \alpha \iota$ -

1) Eine Ergänzung bietet Phot. p. 346 b $23 \mathrm{ff}$. (aus Damaskios): $\dot{\delta} \Pi \alpha \mu \pi \rho \varepsilon_{-}$

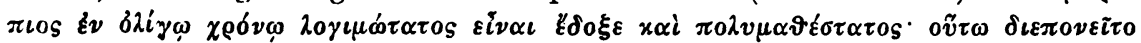

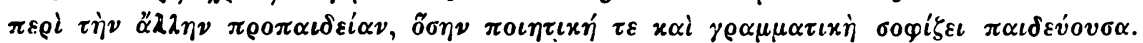

2) Anders der erste Teil des Suidas-Artikels nach Malchos (vgl. Bernhardys An-

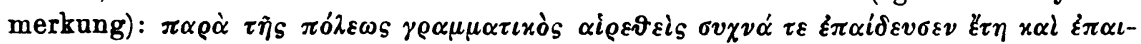

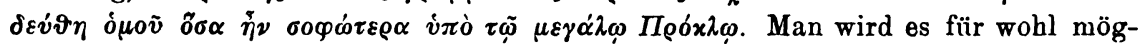
lich halten, daB Pamprepios wirklich unter Proklos Philosophie studierte - daB er für einen Philosophen galt, zeigt das p. 34,10 ff. Erzählte - wenn man bedenkt, wie hart Damaskios auch sonst urteilt und wie er eifersüchtig darüber wacht, dab Leute, die keine Metaphysiker im Sinue des Iamblich, Syrian und Proklos gewesen sind, als Philosophen gerühmt werden. Nicht einmal Aristoteles und Chrysippos entsprechen seinen Anforderungen, und unter den Neueren wird von Hierokles, Hermeias, Domninos, Hypatia betont, daB sie die volle Höhe der Philosophie (im Unterschiede von Polymathie und Spezialwissenschaften) nicht erreicht haben. Vgl. Phot. p. 337 b 27 ff. B. $\$ 36$ W.: (nach Erwähnung von Porphy-

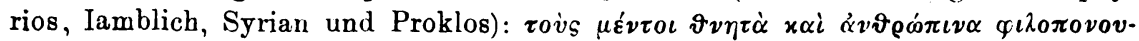

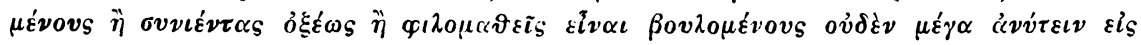

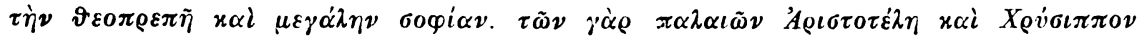

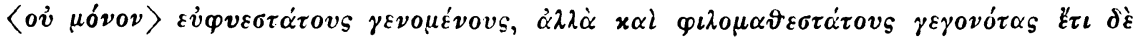

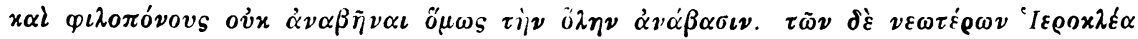

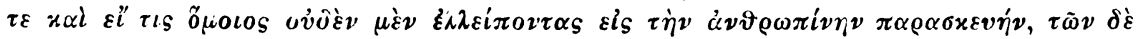

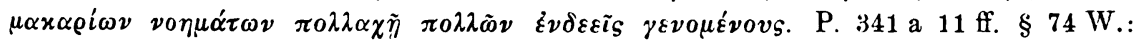

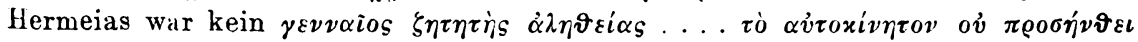


$\left.\delta \varepsilon i \alpha^{1}\right)$ entfaltete er sein ehrgeiziges Streben mit dem Erfolge, daB er den wegen ihrer Polymathie angesehenen Männern Plutarch und Hermeias den Rang ablief.

Nun wird doppelt klar, wie übereilt es ist, wenn Bernhardy den

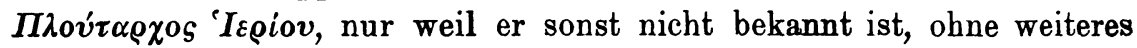

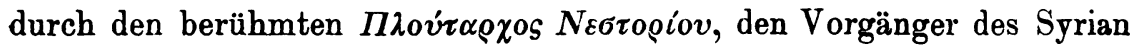
und des Proklos in der Leitung der platonischen Schule Athens, ersetzen will. ${ }^{2}$ ) Dieser Plutarch ist Philosoph und Theurg. Polymathie und hervorragende Beschäftigung mit den propädeutischen Fächern werden ihm nicht nachgerühmt. Wäre er hier genannt, so enthielte die Stelle einen scharfen Widerspruch gegen die vorangehende Angabe, daB Pamprepios mit den Philosophen nicht konkurrierte und überhaupt für Philosophie nicht beanlagt war. Dazu kommt eine chronologische Schwierigkeit. Der Sohn des Nestorios starb nach Marinos' Leb. d. Prokl. 12, als Proklos ungefähr 22 Jahre alt war. Der Plutarch des Suidas aber muB, das geht aus dem Zusammenhange klar hervor, mit dem auf der Höhe seines Ruhmes stehenden Proklos gleichzeitig gelebt haben. Noch viel entscheidender aber widerspricht die Chronologie des Pamprepios der Annahme Bernhardys. Pamprepios hat nicht nur noch beim Aufstande des Markianos in Zenons fünftem Regierungsjahre $^{3}$ ) (478/9) nach Suid. p. 33,5 f. als energischer Berater des Illus eine Rolle gespielt, sondern nach Eustathios p. 359, $26 \mathrm{ff}$. Dind., Theophan. p. 129, $29 \mathrm{ff}$. de Boor auch die Erhebung des Leontios im Juni des zehnten Regierungsjahres (484) miterlebt - nach dem Malalasexzerpt Hermes 6 (1872) S. 372 ernannte ihn Leontios bei der Verteilung der Ämter zum Magistros - und war an den Ereignissen der folgenden vier Jahre aktiv beteiligt. Setzen wir selbst den unwahrscheinlichen Fall, daB Pamprepios hei der Erhebung des Leontios und seiner eigenen Er-

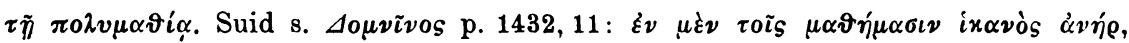

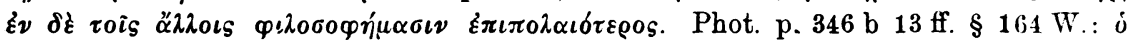

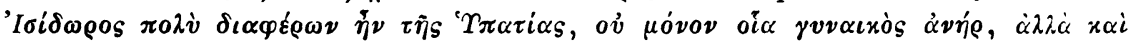

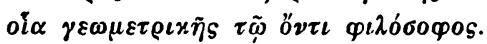

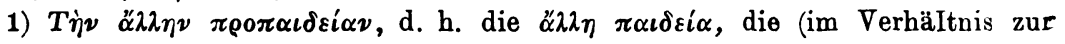

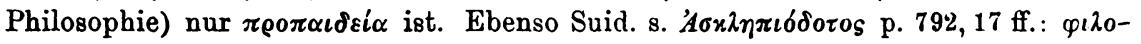

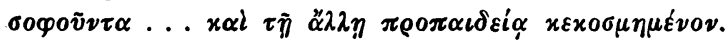

2) $\mathrm{Zu}$ 'Iepiov bemerkt er im Apparat: „Mira depravatio: nam reponendum

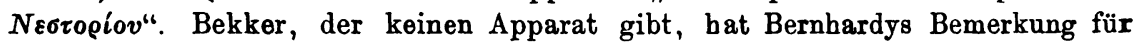
wichtig genug gehalten, um sie im Texte zu berücksichtigen; er fügt hinter 'Ic९lov

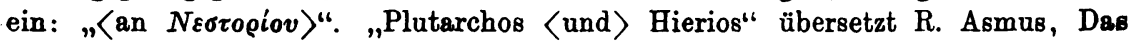
Leben d. Philos. Isid. von Damaskios aus Dam., Leipzig 1911 (Philos. Bibl. Bd. 125) S. 103, der die ganze Stelle sehr frei umgestaltet.

3) Vgl. Theophan. p. 126, 30 de Boor. 
nennung zum Magistros schon $75 \mathrm{Jahre}$ alt gewesen sei, so fällt seine Geburt 409, er war also beim Tode des Plutarch (431/2) 22 oder 23 Jahre alt, so daB nicht anzunehmen ist, er habe den Ruhm des groBen Schulhauptes verdunkelt. Endlich ist es völlig ausgeschlossen, daB Damaskios von dem gefeierten Leiter der Akademie, wie wenn es sich um einen neu einzuführenden Unbekannten handelte, als von Plutarch, dem Sohne des Nestorios, einem Manne aus Athen gesprochen haben sollte. Auch für ihn, wie für Marinos, ist er "der groBe Plutarch" (Suid. s. 'H l $\alpha_{S}$ p. 811,9 f.). Den „heiligen Pl." nennt er ihn dubit. et sol. II

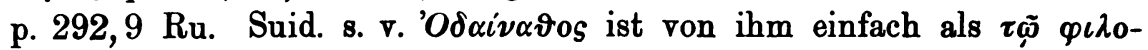

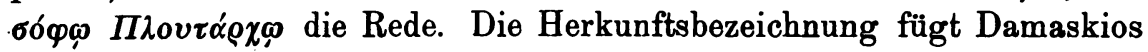
bei Said. s. $\Delta o \mu \nu i v o s$ p. 1432, 20 hinzu $^{1}$ ), wo es auf die athenische Abstammung des Plutarch im Gegensatze zu der syrischen des Domninos ankommt. Aber auch hier heiBt es: „Plutarch der Athener“, und nicht "Plutarch, ein Mann aus Athen".

Ein Plutarch, Sohn des Hierios, ist, wenn er auch sonst nicht genannt wird, doch leicht zu erschlieBen. Der bei Phot. bibl. cod. 242 p. 342 a 16 B. $\S 88 \mathrm{~W}$. erwähnte Hierios war Sohn des Plutarch, ohne Zweifel, da von Plutarch schlechthin die Rede ist, des "GroBen“, was auch seiner Zeit stimmt, da Hierios unter Proklos studierte. Ein Sohn von ihm wird nach dem Grobvater Plutarch geheißen haben. Chronølogisch fügt sich alles aufs beste. Proklos war vielleicht schon 438 in Amte des Diadochos ${ }^{2}$ ) und sicher wirkte er als Lehrer noch wenig $\rightarrow$ Jahre vor seinem ins Jahr 485 fallenden Tode. Seine Wirksamkeit dauerte so lange, daB ihn drei Generationen als Lehrer sehen konnten. Archiadas, der Enkel des Diadochen Plutarch (Mar. v. Procl. 12, vgl. 17), war Mitschüler und Schüler des Proklos (Mar. 17). Dessen Tochter Asklepigeneia heiratete Theagenes (Mar. 29). Dieser hatte, wie sich mit guten Gründen annehmen läßt, den Proklosschüler (Mar.

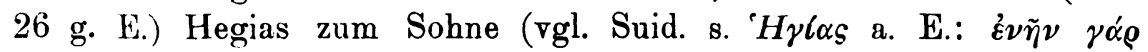

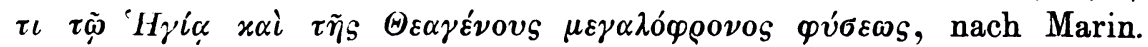
26 g. E. war er vornehmer Abkunft; auf Verwandtschaft mit dem groBen Plutarch deutet Suid. s. 'H l $\alpha \mathrm{s}$ Anf.; ein Sohn des Hegias hieB Archiadas nach Suid. s. Evंrcivıos, Phot. p. 349 a 24 [Damasc. $\S 222 \mathrm{~W}].) .^{3}$ ) Wenn also hier GroBvater und Enkel Proklos zum Lehrer hatten, so steht nichts im Wege, daB Hierios (nach Damask. bei Phot.

1) Ob hier übrigens wirklich der athenische Diadochos gemeint ist, läBt sich nicht mit Sicherheit ausmachen. 1)ie Wahrscheinlichkeit spricht dafür.

2) Vgl. Zeller, Philos. d. Gr. III $2^{4}$ S. 819 , Anm. 4 zu S. 818.

3) Vgl. über diese Verwandtschaftsverhältnisse auch Zeller, Philos. d. Gr. III $2^{4}$ S. 899 Anm. 5.

Byzant. Zeitschrift XXI 3 u. 4. 
p. 342 a 16 ff. B. \& 88 W.) Proklos' Schüler war ${ }^{1}$ ) und sein Sohn Plutarch noch gleichzeitig mit Proklos in Athen lebte und wirkte. Verlegen wir sein Wirken, wie es der Wahrscheinnlichkeit entspricht, in die späteren Jahre des Proklos, so konnte Pamprepios immer noch zu gleicher Zeit mit ihm in Athen anwesend sein. Nach Suid. s. v. 31, 18 hat Pamprepios lange Jahre in Athen unterrichtet. Erst unter Zenon (474-491) ist er in Byzanz nachweisbar. Der Grund seines Scheidens aus Athen war nach Suid. p. 31, 20 der Konflikt mit einem gewissen

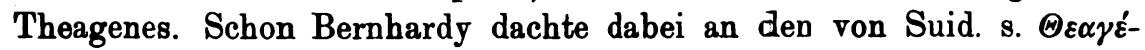
$\nu \eta s$ besprochenen einfluBreichen athenischen Archonten und römischen Senator, denselben, der soeben als Vater des Hegias erwähnt wurde. Ein mächtiger Mann muB es gewesen sein ${ }^{2}$ ), und dab er den $\gamma \rho \alpha \mu \mu \alpha^{\prime}-$ $\tau \omega \nu \delta \iota \delta \alpha ́ \sigma x \alpha \lambda$ os brüskierte, stimmt ganz zu der hochfahrenden Art, die Suid s. $\Theta \varepsilon \alpha \gamma$. beschrieben ist und die auch eine Trübung des Verhältnisses zum Neuplatoniker Marinos herbeiführte. Wer Möglichkeiten ausspinnen will, mag sich vorstellen, daB bei dem Zerwürfnis zwischen Theagenes und Pamprepios eben die von Damaskios bei Suidas berichtete Rivalität des Pamprepios mit Plutarch, einem Verwandten des Theagenes, eine Rolle spielte.

Halle a. S.

Karl Praechter.

1) Und zwar gehörte er als Sohn des Plutarch wohl der älteren Schülergeneration an.

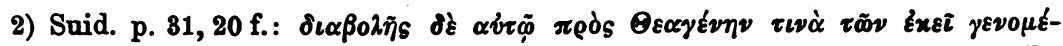

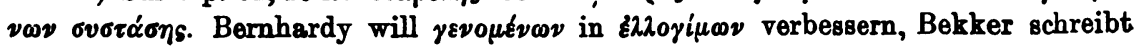
$\delta v \nu \alpha \mu \varepsilon v \omega \nu$. Ich vermute $\delta v \nu \alpha \tau \tilde{\omega} \nu$ $\gamma \varepsilon v 0 \mu \varepsilon v \omega \nu$. 